\title{
Hyoid Bone Position as an Etiological Factor in Mandibular Divergence and Morphology
}

\author{
Tiffany Pei-Jou Chen', Falon Rodhisky², Shuying Sue Jiang ${ }^{3}$, Thomas J. Cangialosi ${ }^{4}$ \\ ${ }^{1}$ Private Practice of Orthodontics, Manalapan, NJ, USA \\ ${ }^{2}$ Private Practice of Orthodontics, Hope Mills, NJ, USA \\ ${ }^{3}$ Rutgers School of Dental Medicine, Newark, NJ, USA \\ ${ }^{4}$ Department of Orthodontics, School of Dental Medicine, Rutgers University, Newark, NJ, USA \\ Email: cangiatj@sdm.rutgers.edu
}

How to cite this paper: Chen, T.P.-J., Rodhisky, F., Jiang, S.S. and Cangialosi, T.J. (2022) Hyoid Bone Position as an Etiological Factor in Mandibular Divergence and Morphology. Open Journal of Orthopedics, $12,10-25$.

https://doi.org/10.4236/ojo.2022.121002

Received: November 23, 2021

Accepted: January 21, 2022

Published: January 24, 2022

Copyright $\odot 2022$ by author(s) and Scientific Research Publishing Inc. This work is licensed under the Creative Commons Attribution International License (CC BY 4.0).

http://creativecommons.org/licenses/by/4.0/ Open Access

\begin{abstract}
Objectives: The objective is to determine whether there are differences in the position of the hyoid bone at rest in natural head position in subjects with mandibular hyperdivergence and to evaluate whether there are differences in hyoid position and antegonial notch depth in mandibular hyperdivergent males and females. Methods and Materials: This is a retrospective cohort study involving a review of lateral cephalometric radiographs of 45 adult men and women with mandibular hyperdivergency. Hyperdivergency was determined by cephalometric ranges of: SN-GoGn as least $+2 \mathrm{SD}$ from normal, $\mathrm{Y}$-axis, PP-GoGN, and gonial angle greater than $+1 \mathrm{SD}$ from normal. A group of 45 normodivergent adults served as a control, with cephalometric ranges of: SN-GoGn within $\pm 1 \mathrm{SD}$ of normal, with only one measurement of the other three between +1 and +1.5 standard deviations. A custom digital cephalometric analysis, the Hyoid Analysis, was designed, to measure the vertical and horizontal position and inclination of the hyoid and the antegonial notch depth. Results: In hyperdivergent subjects, the posterior aspect of the hyoid is located lower and more posterior, compared to the control group, while there is no difference in position of the anterior surface of the hyoid and the antegonial notch is $0.6 \mathrm{~mm}$ deeper. In males, the posterior aspect of hyoid is lower by $8.5 \mathrm{~mm}$, while the anterior surface is located $9.0 \mathrm{~mm}$ lower. In males, the hyoid is inclined more steeply than in females by 4.4 degrees and the antegonial notch is deeper than in females by $0.6 \mathrm{~mm}$. Conclusions: There are differences in hyoid bone position and mandibular morphology in hyperdivergent subjects compared to normodivergent subjects and in males compared to females.
\end{abstract}

\section{Keywords}

Hyoid Bone, Mandibular Divergence, Orthopedic, Surgery 


\section{Introduction}

Patients with a hyperdivergent mandibular growth pattern often present a challenge for orthodontic treatment, due to a dental open bite tendency that may be complicated by underlying issues such as allergies, lymphatic tissue hypertrophy, muscular hypotonicity, syndromes, and neurologic disorders. Although some open bite cases have an environmental etiology, such as digit-sucking or forward posturing and thrusting of the tongue, other factors may be genetic, such as over-eruption of posterior teeth, a vertical growth pattern of the mandible and airway obstruction [1] [2]. Many cases of mandibular hyperdivergency are established early in growth and do not improve over time [3]. The mandibular morphology in these hyperdivergent patients is distinct: the anterior corpus of the mandible is bent downward and there is strong antegonial notching [4], but exactly how the mandible develops in this manner is unknown.

The etiology of vertical discrepancy is multifactorial and it has been postulated that hyperdivergency may be related to the position of the hyoid bone and the interaction of the suprahyoid muscles with the muscles of mastication [5]. The major muscles of mastication, including the temporalis, masseter, medial and lateral pterygoid have various origins on the cranium but all are inserted at or near the angle of the mandible. When activated, these muscles exert a force elevating the mandible from the posterior region. Conversely, the suprahyoid muscles, including the mylohyoid, geniohyoid and anterior belly of the digastric muscles, have their origins on the anterior corpus of the mandible and insert on the body of the hyoid bone. When activated, these muscles have a depressing effect on the anterior corpus of the mandible. It has been postulated that the interaction between these elevator and depressor muscles on the mandible may create an envelope of function which is directed through the antegonial area, creating a deep notch as the anterior corpus of the mandible is bent downward) [6].

One theory suggests that the growth at the mandibular condyle stops in hyperdivergent patients, while the masseter and medial pterygoid muscle that insert at the gonial angle continue to grow. This muscle growth causes bone deposition at the gonial angle and prevents the resorption that is typical to this area. The cumulative effect is that a notch occurs just anterior to the gonial area [7].

Another theory combines the previous one with elements from the functional matrix hypothesis, which states that the amount of condylar growth indirectly affects the direction of mandibular rotation and the resulting antegonial notch depth. If the growth of the condyle is less than the vertical growth at the midface and alveolus, the mandible rotates downward and backward. The anterior part of the mandible is pushed down into the soft tissue matrix, resulting in resorption below the symphysis while the posterior part of the corpus is lifted up from the soft tissue matrix, stretching the periosteum, and causing apposition to occur below the angle of the mandible. This forms the antegonial notch in hyperdiver- 
gent subjects with a short ramus height [8].

Since the hyoid bone has multiple muscular connections to the mandible and the tongue, it has also been postulated that the hyoid may also have a role in the development of mandibular morphology. The hyoid bone, having no bony connection itself, is stabilized mainly by muscles [9]. The suprahyoid muscles consist of the digastric, stylohyoid, geniohyoid, and mylohyoid muscles, while the infrahyoid muscles consist of the sternohyoid, thyrohyoid, sternothyroid and omohyoid muscles which insert on the sternum, the thyroid cartilage and the scapula [5] [10]. Thus, it is possible that the position of the hyoid bone may be related to mandibular morphology through its strong muscular connections.

Determining the position of the hyoid bone is difficult since it is mobile. Slight changes in the position of the hyoid bone may occur with changes in posture, head position, tongue position, and function such as swallowing, mastication and respiration [11] [12] [13] [14] [15]. Additionally, its position changes over time with growth, moving downward and forward [16] [17] and increasing with age [18] [19].

However, the resting position of the hyoid has been found to be stable [20]. Typically, it is vertically positioned anterior to the lower portion of the third cervical vertebra [21] at a fixed distance from that vertebra [22]. This stable position is thought to be due to the necessity of maintaining a patent airway [18]. This has been found to be evident even after a surgical procedure to reposition the mandible posteriorly [22]. Additionally, the hyoid position is stable even in patients who have tongue thrust and mouth breathing habits [20]. In these cases, the resting hyoid position has been found to be highly reproducible on a lateral cephalometric radiograph in natural head position [23].

Some studies have compared the hyoid bone position in hyperdivergent subjects with normodivergent and/or hypodivergent subjects on lateral cephalometric radiographs and came to conflicting conclusions. Bibby [5], Subtelny [24], and Jena [25] all found no difference in hyperdivergent subjects as compared to normodivergent subjects. On the other hand, Haralabikis [26] found significant differences in the horizontal position and inclination of the hyoid but no differences in the vertical position. It was found that the hyoid was more posterior and more steeply inclined in hyperdivergent subjects. Additionally, Joseph [27] found that the hyoid is more inferior in hyperdivergent subjects, while Tumpkin [2] and Opdebeeck [28] found that the hyoid is more posterior in hyperdivergent subjects.

There have been conflicting findings on whether there are differences in hyoid position in males compared to females. Some studies have found there are no differences in gender [5] and others have found a difference only when relating the hyoid to the cervical vertebrae [29]. This differenced is appears after normalizing for head size [15]. Others have found that there are differences but these studies are not in agreement. For instance, Adamidis [14] found that the anterior point of the hyoid is higher in boys aged 10 - 13 years than girls of the same age, while Arslan [29] found that the hyoid is more inferior and anterior in males. 
Since there is little definitive data correlating hyoid position and antegonial notch depth with mandibular morphology, a cephalometric analysis was designed to determine the anatomical differences between hyperdivergent and normodivergent subjects and to assess for sexual dimorphisms.

\section{Objectives of Study}

The objectives of this study are to determine whether there are differences in the position of the hyoid bone at rest in natural head position, vertically, horizontally or in axial inclination. Secondly, what is the effect of hyoid bone position on the antegonial notch depth in subjects with mandibular hyperdivergency as compared to normodivergent subjects? Thirdly, to evaluate whether there are differences in hyoid position and antegonial notch depth between males and females.

\section{Null Hypothesis}

The null hypothesis states that there are no differences in hyoid bone position or antegonial notch depth in hyperdivergent subjects as compared to normodivergent subjects. Second, there are no morphological differences in hyoid bone position or antegonial notch depth in non-growing males as compared to nongrowing females.

\section{Materials and Methods}

\subsection{Subject Selection}

This is a retrospective cohort study involving a review of Lateral cephalometric radiographs. All records taken prior to September 11, 2017 at Rutgers University, School of Dental Medicine Department of Orthodontics were searched in Dolphin Imaging. In order to define the hyperdivergent group, cephalometric measurement norms from Cangialosi [30] were used for the angles SN-GoGn, $\mathrm{Y}$-axis, SN-PP, PP-GoGn, and gonial angle and the ratio, UFH/LFH ratio (see Table 1).

Inclusion criteria were defined as: male and female patients with all permanent teeth erupted except third molars, a Cervical Vertebral Maturation Index of 5 or 6 (indicating a non-growing individual), and high quality lateral cephalometric radiographs taken in natural head position with the hyoid bone clearly depicted. Exclusion criteria were defined as: patients with clefts or craniofacial syndromes, major facial or mandibular asymmetries, cervical vertebrae anomalies, missing permanent teeth (excluding third molars), a history of treatment with implants, orthodontics or orthognathic surgery, or poor-quality cephalometric radiographs. No other socioeconomic data was included.

The most hyperdivergent subjects had the following characteristics which ultimately became the cephalometric criteria for the experimental group: an SN-GoGN angle of at least +2 standard deviations from normal, with three other measurements ( $\mathrm{Y}$-axis, PP-GoGN, and gonial angle) at least greater than +1 
Table 1. Norms and standard deviations of cephalometric measurements from Cangialosi [30].

\begin{tabular}{clcc}
\hline \multicolumn{4}{c}{ Norms and Standard Deviation Values for Subject Selection } \\
\hline Angle & \multicolumn{1}{c}{ Definition } & Normal & SD \\
\hline Sn-GoGn & $\begin{array}{l}\text { Angle between the SN plane and the mandibular plane } \\
\text { defined as Go-Gn }\end{array}$ & $29.8^{\circ}$ & \pm 5.5 \\
SN-PP & $\begin{array}{l}\text { Angle between the SN plane and palatal plane defined } \\
\text { as ANS-PNS }\end{array}$ & $8.2^{\circ}$ & \pm 3.3 \\
Y-axis & $\begin{array}{l}\text { Angle between SN plane and line connecting Sella and } \\
\text { Gnathion }\end{array}$ & $66.5^{\circ}$ & \pm 3.7 \\
PP-GoGn & $\begin{array}{l}\text { Angle between the palatal plane defined as ANS-PNS } \\
\text { and mandibular plane defined as Go-Gn }\end{array}$ & $21.9^{\circ}$ & \pm 5.6 \\
UFH/LFH & $\begin{array}{l}\text { Ratio between upper facial height and lower facial } \\
\text { height }\end{array}$ & 0.812 & \pm 0.082 \\
Ar-Go-Me & Gonial angle & $123.9^{\circ}$ & \pm 5.4 \\
\hline
\end{tabular}

standard deviation from normal (Table 2). Ultimately, 45 subjects, including 10 males and 35 females, were chosen for the hyperdivergent group from 522 subjects.

A similar process was used to choose the control group. Lateral Cephalometric radiographs in Dolphin Imaging were searched according to the same inclusion and exclusion criteria. The same Cephalometric values for all subjects in the control group were within 1 standard deviation from normal.

The records with the cephalometric values closest to normal were chosen, resulting in the following cephalometric criteria: SN-GoGn within \pm 1 standard deviation from normal, with only one out of the other three measurements in the acceptable range of +1 and +1.5 standard deviations (Table 3 ). Ultimately, 45 subjects, including 18 males and 27 females, were chosen for the control group from 2891 subjects.

The hyperdivergent group included 9 males and 36 females, while the normodivergent group included 18 males and 27 females.

Subjects in both groups were then arranged in order of increasing SN-GoGn value and assigned a coded number, ranging from $\mathrm{C} 1$ - $\mathrm{C} 45$ for the normodivergent group and $\mathrm{H} 1-\mathrm{H} 45$ for the hyperdivergent group.

\subsection{Hyoid Analysis}

A digital cephalometric analysis (Hyoid Analysis) was designed in Dolphin Imaging to measure the hyoid position and depth of the antegonial notch. Two points were chosen to determine hyoid position. H-point is defined as the most superior and anterior point on the anterior curvature of the body of the hyoid. G-point is defined as the most posterior point on the greater cornu of the hyoid (the posterior end of the tubercle). In cases where the hyoid was captured at an 
Table 2. Standard deviation and value ranges of cephalometric measurements used to define the hyperdivergent group.

\begin{tabular}{ccc}
\hline \multicolumn{2}{c}{ Cephalometric Requirements for Hyperdivergent Group } \\
\hline Angle & Minimum SD & Minimum Value \\
\hline Sn-GoGn & $>2$ & $>40.8^{\circ}$ \\
Y-axis & $>1$ & $>70.2^{\circ}$ \\
PP-GoGn & $>1$ & $>27.5^{\circ}$ \\
Ar-Go-Me & $>1$ & $>129.3^{\circ}$ \\
\hline
\end{tabular}

Table 3. Standard deviation and value ranges of cephalometric measurements used to define the normodivergent group. Required measurements included: SN-GoGn in the ideal range, with only up to one out of the other three in the acceptable range.

\begin{tabular}{ccccc}
\hline \multicolumn{4}{c}{ Cephalometric Requirements for Normodivergent Group } \\
\hline \multicolumn{2}{c}{ Ideal Values } & \multicolumn{2}{c}{ Acceptable Values } \\
\hline Angle & SD Range & Value Range & SD Range & Value Range \\
\hline SN-GoGn & -1 to +1 & $24.3^{\circ}$ to $35.3^{\circ}$ & - & - \\
Y-axis & -1 to +1 & $62.8^{\circ}$ to $70.2^{\circ}$ & +1 to +1.5 & $70.2^{\circ}$ to $72.05^{\circ}$ \\
PP-GoGn & -1 to +1 & $16.3^{\circ}$ to $27.5^{\circ}$ & +1 to +1.5 & $27.5^{\circ}$ to $30.3^{\circ}$ \\
Ar-Go-Me & -1 to +1 & $118.5^{\circ}$ to $129.3^{\circ}$ & +1 to +1.5 & $129.3^{\circ}$ to $132^{\circ}$ \\
\hline
\end{tabular}

angle on the lateral cephalometric radiograph, resulting in two distinct greater cornu, G-point was placed at the midpoint between the two posterior ends of the tubercles.

To measure the vertical position of the hyoid, a reference plane was drawn: the palatal plane, defined as ANS-PNS. The perpendicular distance from the palatal plane to H-point and G-point were then measured in millimeters. A positive value indicated the points were superior to the reference plane.

To measure the horizontal position of the hyoid, two reference planes were drawn perpendicular to the Frankfort Horizontal plane. One plane was drawn at sella (Sperp), and the other was drawn at the most posterior point on the outline of the pterygomaxillary fissure (PTMperp) according to the method by Jena [25]. The perpendicular distances from G-point to Sperp and from H-point to PTMperp were measured in millimeters. A positive value indicated the points were anterior to the reference plane.

To measure the axial inclination of the hyoid, the angle between the hyoid plane (H-axis, defined as a plane from H-point to G-point) and a horizontal plane seven degrees at Nasion to the $\mathrm{SN}$ line, was measured in degrees.

To measure the depth of the antegonial notch, one reference plane was drawn: the mandibular plane, (defined as a line tangent to the lower border of the mandible). Antegonial notch depth was then measured as the perpendicular distance from the deepest portion of the antegonial notch (point AGN) to the mandibular tangent plane, in millimeters. See Figure 1 for a summary of all 


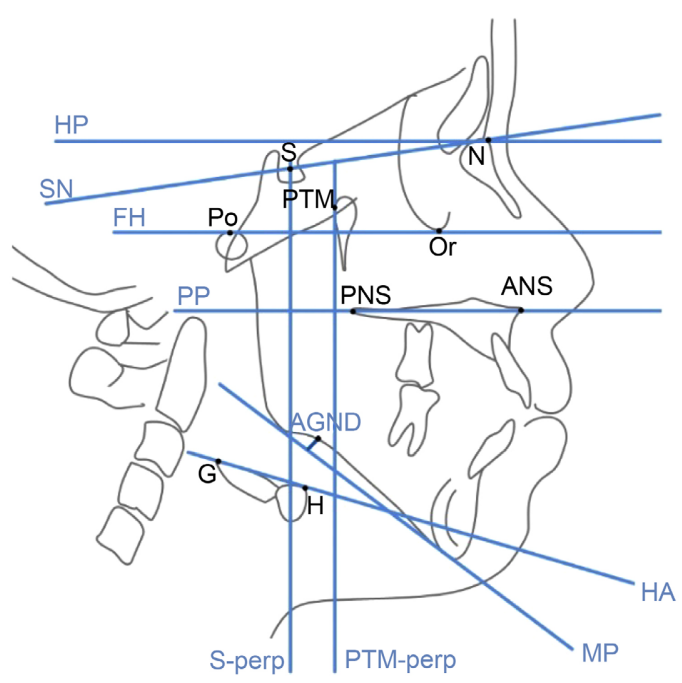

\begin{tabular}{|l|l|}
\hline \multicolumn{2}{|l|}{ Cephalometric Points } \\
\hline Po & Porion \\
\hline Or & Orbitale \\
\hline S & Sella \\
\hline N & Nasion \\
\hline PTM & $\begin{array}{l}\text { Most posterior point of } \\
\text { pterygomaxillary fissure }\end{array}$ \\
\hline ANS & Anterior nasal spine \\
\hline PNS & Posterior nasal spine \\
\hline $\begin{array}{l}\text { H-point } \\
\text { (H) }\end{array}$ & $\begin{array}{l}\text { Most superior and anterior point } \\
\text { on the anterior curvature of the } \\
\text { body of the hyoid }\end{array}$ \\
\hline $\begin{array}{l}\text { G-point } \\
\text { (G) }\end{array}$ & $\begin{array}{l}\text { Most posterior point on the } \\
\text { greater cornu of the hyoid (the } \\
\text { posterior end of the tubercle) }\end{array}$ \\
\hline AGND & $\begin{array}{l}\text { Antegonial notch depth - Deepest } \\
\text { portion of the antegonial notch }\end{array}$ \\
\hline
\end{tabular}

\begin{tabular}{|l|l|}
\hline Reference Plane & Definition \\
\hline Frankfurt Horizontal (FH) & Po-Or \\
\hline Horizontal Plane (HP) & 7 degrees above SN at nasion \\
\hline Palatal Plane (PP) & ANS-PNS \\
\hline Sperp & Vertical line from S, perpendicular to FH \\
\hline PTMperp & Vertical line from PTM \\
\hline Hyoid Axis (HA) & H-point - G-point \\
\hline Tweed Mandibular Plane (MP) & Line tangent to the lower border of mandible \\
\hline
\end{tabular}

\begin{tabular}{|l|l|l|}
\hline \multicolumn{3}{|c|}{ Hyoid Analysis Measurements } \\
\hline Measurement & \multicolumn{1}{c|}{ Definition } & \multicolumn{1}{c|}{ Unit } \\
\hline G-PP & Perpendicular distance from G-point to palatal plane & Millimeters \\
\hline H-PP & Perpendicular distance from H-point to palatal plane & Millimeters \\
\hline G-Sperp & Perpendicular distance from G-point to Sperp & Millimeters \\
\hline H-PTMperp & Perpendicular distance from H-point to PTMperp & Millimeters \\
\hline Haxis-HP & Angle between hyoid axis and horizontal plane & Degrees \\
\hline Antegonial notch depth & $\begin{array}{l}\text { Perpendicular distance from deepest portion of } \\
\text { antegonial notch to the mandibular tangent plane }\end{array}$ & Millimeters \\
\hline
\end{tabular}

Figure 1. Tracing of Landmarks, reference planes, and measurements used in the Hyoid analysis. Ante-Gonial Notch Depth (AGND).

cephalometric measurements made.

All cephalometric radiographs in each group were traced by the primary examiner (T.C.) and a second examiner (F.R.).

\section{Statistical Analyses}

IBM SPSS version 24 was used forall data analysis. Two-way ANOVA was used 
to determine whether there is a significant difference between the mandibular divergence groups and genders. The significance level was set to $p<0.05$. Intraclass Correlation Coefficient (ICC) was used for inter- and intra-examiner reliability, using a Two-Way Mixed and Consistency model.

\section{Results}

For inter-examiner reliability, both examiners (T.C. and F.R.) were in agreement, as all ICC values were above 0.800 (Table 4). Each examiner waited one month after the initial tracing to retrace the radiographs again. Intra-examiner reliability was good except for G-Sperp which was 0.72 (Table 5).

A summary of all measurements and statisticsis is shown in Table 6. Two-way interaction between mandibular divergence and gender was checked in all models. No significance was found.

G-PP was significant for both the mandibular divergence and gender groups. In hyperdivergent subjects, the posterior aspect of the hyoid was $47.2 \mathrm{~mm}$ below palatal plane, which is $1.3 \mathrm{~mm}$ lower than in the normodivergent group $(\mathrm{p}$-value $=$ 0.029). In males, the posterior aspect of the hyoid is $52.4 \mathrm{~mm}$ below the palatal plane, which is $8.5 \mathrm{~mm}$ lower than in females ( $\mathrm{p}$-value $<0.001$ ).

$\mathrm{H}$-PP was significant only for gender. In males, $\mathrm{H}$-point is $65.7 \mathrm{~mm}$ below the palatal plane, which is $9.0 \mathrm{~mm}$ lower than infemales (p-value $<0.001$ ). There was

Table 4. Inter-examiner reliability for both Examiner 1 and Examiner 2 (completed as part of pilot study).

\begin{tabular}{cl}
\hline \multicolumn{1}{c}{ Inter-Examiner Reliability } \\
\hline Measurement & ICC \\
\hline G-PP & 0.949 \\
H-PP & 0.980 \\
G-Sperp & 0.983 \\
H-PTMperp & 0.993 \\
Haxis-HP & 0.925 \\
AGND & 0.845 \\
\hline
\end{tabular}

Table 5. Intra-examiner reliability for Examiner 1 (completed as part of pilot study).

\begin{tabular}{cl}
\hline \multicolumn{2}{c}{ Inter-Examiner Reliability } \\
\hline Measurement & ICC \\
\hline G-PP & 0.978 \\
H-PP & 0.997 \\
G-Sperp & 0.728 \\
H-PTMperp & 0.924 \\
Haxis-HP & 0.974 \\
AGND & 0.967
\end{tabular}


Table 6. Descriptive statistics for each cephalometric measurement of the Hyoid Analysis, according to gender and group. An asterisk $\left(^{*}\right)$ denotes a significant p-value.

\begin{tabular}{|c|c|c|c|c|c|}
\hline \multicolumn{6}{|c|}{ G-PP } \\
\hline & & $\mathrm{N}$ & Mean & SD & $\mathrm{p}$-value \\
\hline Mandibular & $\mathrm{C}$ & 45 & -45.867 & 6.6374 & \multirow{2}{*}{$0.029 *$} \\
\hline Divergence & $\mathrm{H}$ & 45 & -47.233 & 7.6436 & \\
\hline \multirow{2}{*}{ Gender } & $\mathrm{M}$ & 28 & -52.414 & 7.1194 & \multirow{2}{*}{$<0.001^{\star}$} \\
\hline & $\mathrm{F}$ & 62 & -43.902 & 5.4092 & \\
\hline Interaction & & & & & 0.909 \\
\hline \multicolumn{6}{|c|}{ H-PP } \\
\hline & & $\mathrm{N}$ & Mean & SD & $\mathrm{p}$-value \\
\hline Mandibular & $\mathrm{C}$ & 45 & -59.313 & 7.0098 & \multirow{2}{*}{0.242} \\
\hline Divergence & $\mathrm{H}$ & 45 & -59.620 & 8.2637 & \\
\hline \multirow{2}{*}{ Gender } & $\mathrm{M}$ & 28 & -65.675 & 7.1523 & \multirow{2}{*}{$<0.001^{\star}$} \\
\hline & $\mathrm{F}$ & 62 & -56.663 & 6.0341 & \\
\hline Interaction & & & & & 0.731 \\
\hline \multicolumn{6}{|c|}{ G-Sperp } \\
\hline & & $\mathrm{N}$ & Mean & $\mathrm{SD}$ & p-value \\
\hline Mandibular & $\mathrm{C}$ & 45 & -15.013 & 5.7561 & \multirow{2}{*}{$0.035^{*}$} \\
\hline Divergence & $\mathrm{H}$ & 45 & -18.193 & 6.1654 & \\
\hline \multirow{2}{*}{ Gender } & $\mathrm{M}$ & 28 & -15.404 & 5.7712 & \multirow{2}{*}{0.436} \\
\hline & $\mathrm{F}$ & 62 & -17.145 & 6.2726 & \\
\hline Interaction & & & & & 0.937 \\
\hline \multicolumn{6}{|c|}{ H-PTMperp } \\
\hline & & $\mathrm{N}$ & Mean & SD & p-value \\
\hline Mandibular & $\mathrm{C}$ & 45 & -0.282 & 6.2142 & \multirow{2}{*}{0.328} \\
\hline Divergence & $\mathrm{H}$ & 45 & 1.324 & 8.5217 & \\
\hline \multirow{2}{*}{ Gender } & $\mathrm{M}$ & 28 & -0.646 & 7.1064 & \multirow{2}{*}{0.476} \\
\hline & $\mathrm{F}$ & 62 & 1.048 & 7.6110 & \\
\hline Interaction & & & & & 0.598 \\
\hline \multicolumn{6}{|c|}{ Haxis-HP } \\
\hline & & $\mathrm{N}$ & Mean & SD & $\mathrm{P}$-value \\
\hline Mandibular & $\mathrm{C}$ & 45 & 25.480 & 7.5140 & \multirow{2}{*}{0.269} \\
\hline Divergence & $\mathrm{H}$ & 45 & 28.844 & 9.1646 & \\
\hline \multirow{2}{*}{ Gender } & $\mathrm{M}$ & 28 & 24.071 & 8.3166 & \multirow{2}{*}{$0.036^{*}$} \\
\hline & $\mathrm{F}$ & 62 & 28.558 & 8.2767 & \\
\hline Interaction & & & & & 0.524 \\
\hline \multicolumn{6}{|c|}{ AGND } \\
\hline & & $\mathrm{N}$ & Mean & SD & $\mathrm{p}$-value \\
\hline Mandibular & $\mathrm{C}$ & 45 & 1.764 & 0.8650 & \multirow{2}{*}{$<0.001^{\star}$} \\
\hline Divergence & $\mathrm{H}$ & 45 & 2.413 & 1.0714 & \\
\hline \multirow{2}{*}{ Gender } & $\mathrm{M}$ & 28 & 2.493 & 1.0597 & \multirow{2}{*}{$<0.001^{\star}$} \\
\hline & $\mathrm{F}$ & 62 & 1.906 & 0.9578 & \\
\hline Interaction & & & & & 0.252 \\
\hline
\end{tabular}


no significant difference between hyperdivergent and normodivergent subjects.

G-Sperp was significant only for the mandibular divergence group. In hyperdivergent subjects, G-point is $18.2 \mathrm{~mm}$ posterior to Sperp, which is $3.2 \mathrm{~mm}$ more posterior than in normodivergent subjects $(\mathrm{p}$-value $=0.035)$. There was no significant difference between genders.

H-PTMperp was not significant for eithermandibular divergenceor gender.

$\mathrm{H}$-axis-HP was significant only for gender. In males, the hyoid is inclined at a mean of $24.1^{\circ}$, which is $4.4^{\circ}$ higher than in females ( $p$-value $=0.036$ ). There was no significant difference between hyperdivergent and normodivergent subjects.

AGND was significant for both mandibular divergence and gender. In hyperdivergent subjects, the antegonial notch is a mean of $2.4 \mathrm{~mm}$ deep, which is 0.6 $\mathrm{mm}$ deeper than in normodivergent subjects ( $\mathrm{p}$-value $<0.001$ ). In males, the antegonial notch is a mean of $2.5 \mathrm{~mm}$ deep, which is $0.6 \mathrm{~mm}$ deeper than in females ( $\mathrm{p}$-value $<0.001$ ).

\section{Discussion}

\subsection{Hyoid Position}

Cranial base landmarks have been traditionally chosen as reference points from which to measure the vertical and horizontal position of the hyoid bone, since multiple studies have found that the hyoid is consistent in location when compared to local landmarks, including the cervical vertebrae, pharynx, and cervical spine [18] [26] [31]. The reason for this is thought to be due to the necessity of maintaining a consistently patent airway [18] [22] [28]. Therefore, landmarks further away may reveal more differences in hyoid position.

Although the mandible is further away than the cervical vertebrae and multiple studies have used it as a reference plane from which to measure hyoid bone position [5] [10] [14] [26], the mandible was not used in this study for multiple reasons. First, it has been found that the hyoid bone may move in close conjunction with the mandible due to the connecting muscles and ligaments, resulting in minimal differences when using the mandibular plane as a reference for hyoid position [26]. Second, the differences in mandibular plane steepness are being tested in this study. Therefore, utilizing the mandible as a reference frame may affect the results and prevent accurate assessment of the hyoid position.

The data from this study shows that the posterior aspect of the hyoid (G-point) is indeed in a different position in hyperdivergent subjects as compared to normodivergent subjects. In hyperdivergent subjects, G-point is located $1.3 \mathrm{~mm}$ lower and $3.2 \mathrm{~mm}$ more posterior than in normodivergent subjects. There was not a significant difference in the vertical or horizontal position of $\mathrm{H}$-point or the inclination of the hyoid.

These results are similar to the findings by Jena [25] in which the horizontal position, vertical position and inclination of the hyoid in hyperdivergent and normodivergent subjects are comparable as measured at H-point. However, the studies differ in that no measurements were completed at G-point, so that data from this study including G-point cannot be compared. 
Haralibikis [26] also studied the hyoid position in hyperdivergent and normodivergent subjects and separated subjects based on gender. Overall, significant differences in the horizontal position and inclination of the hyoid were found, while there were no differences in the vertical position. H-point was found to be significantly more posterior in open bite subjects than in normal subjects when measured from PTR $\perp$ FH (the equivalent of PTM-perp) and Po $\perp$ $\mathrm{FH}$. This is in direct contrast to the results of this study, which found no difference between hyperdivergent and normodivergent subjects. It was also found that hyperdivergent subjects had a more steeply inclined hyoid when measured from the palatal plane and basion-nasion plane. This is in contrast to this study, which found that there is no difference in hyoid inclination. However, the data cannot be directly compared, since the reference planes used are different. Lastly, there were no vertical differences found between hyperdivergent and normodivergent subjects as measured from $\mathrm{H}$-point, which is again similar to our findings.

The findings of this study do not agree with studies which found that the hyoid at $\mathrm{H}$-point is more inferior or more posterior in hyperdivergent subjects. For instance, Joseph [27] measured the perpendicular distance from $\mathrm{H}$-point to the mandibular plane and found that the hyoid is more inferior in hyperdivergent subjects than normodivergent subjects, which is in conflict with multiple studies that have found that the hyoid moves in conjunction with the mandible.

Our data shows that there is a significant difference in hyoid position with regard to gender. In males, the entire hyoid is located at a lower position while the hyoid is more steeply inclined. This contradicts studies that have found no sexual dimorphism in hyoid bone position, including Bibby [5], Subtelny [24], and Andersen [31]. However, these studies all recruited young, growing children as subjects. Growth may affect the conclusions of these studies, since the hyoid has been found to move forward and downward with growth [16] [17] and increasing age [18] [19].

It has been found that hyperdivergent patients have shorter cranial bases [32] [33]. The vertical discrepancy could be that the palatal plane of hyperdivergent patients is somehow higher in the posterior region, causing the distance from G-point to the palatal plane to be increased but no difference in the distance to H-point. However, this would contradict Nahoum's findings that patients with open bites tend to be hyperdivergent and may have a tipped up palatal plane [34] [35] which would actually increase the distance to H-point instead of G-point.

However, Haralabikis [26] also measured the distance from H-point to the palatal plane and found that this value was increased in males. It was determined that this occurred because the palatal plane was tipped upward. Measurements were not made from G-point, so this data cannot be compared. It is likely that these differences are due to head size differences between males and females.

\subsection{Antegonial Notch Depth}

In this study, the antegonial notch was found to be $0.6 \mathrm{~mm}$ deeper in hyperdi- 
vergent subjects, which is statistically significant, and several studies [4] [36] [37] [38] support the finding that hyperdivergent subjects have a deeper antegonial notch.

Singer [4] conducted a study in which cephalometric measurements of subjects with deep antegonial notches (greater than $3 \mathrm{~mm}$ in depth) were compared with subjects with shallow antegonial notches (less than $1 \mathrm{~mm}$ ). They found that those with deeper notches are more hyperdivergent (SN-MP is increased by 9.62 degrees), have a larger gonial angle (Ar-Go-Gn is increased by 6.14 degrees), are more retrusive (SNB is decreased by 3.96 degrees) and have shorter mandibles (Go-Gn length is decreased by $8.13 \mathrm{~mm}$, while Ar-Gn is decreased by $3.04 \mathrm{~mm}$ ), and (ANS-Me is increased by $5.15 \mathrm{~mm}$ ). Lambrechts [36] also found that those subjects with deeper notches had steeper mandibular planes, more retrusive chins, longer anterior facial heights, and larger gonial angles.

Davidovitch [37] found that antegonial notch depth is smallest in hypodivergent subjects, increases in normodivergent subjects, and is largest in hyperdivergent subjects. This supports the findings that hyperdivergent subjects have deeper antegonial notches than normodivergent subjects. He also found that males have a deeper antegonial notch than females in hypodivergent, normodivergent, and hyperdivergent groups. Mangla [38], who measured the antegonial notch depth using the same method as this study found no sexual dimorphism in antegonial notch depth.

Stronger muscular activity may account for increased antegonial notch depth in males. Notch depth has been found to be correlated with increased masseter muscle activity which may increase bone deposition at the gonial angle, thereby deepening antegonial notch depth [39]. Therefore, it appears that mandibular morphology is largely dependent on the action and interaction of the muscles which surround it.

\subsection{Limitations}

The sample size of this study was relatively small due to limitation of the sample to meet relatively strict cephalometric criteria for hyperdivergency. Additionally, the number of female and male subjects was not equal. However, this study did find significant sexual dimorphism between hyoid bone position and antegonial notch depth. Therefore, it would be beneficial in future studies to attain the same number of male and female subjects and to split the pooled subjects into four groups: male hyperdivergent subjects, male normodivergent subjects, female hyperdivergent subjects, and female normodivergent subjects, as Haralabikis [26] did in his study. This would allow the comparison of hyperdivergent subjects to normodivergent subjects in each gender and would help correct for any gender issues that may have affected the data in this study, such as head size differences between males and females.

\subsection{Clinical Significance and Future Directions}

Further research into the etiology of mandibular hyperdivergency will ultimately 
help clinicians' better understanding of the anatomical limitations of orthodontic treatment. This study indicates that there are indeed anatomical differences outside the mandible, which are related to its morphology. If the precise etiology can be more clearly determined, it may be possible to better determine which patients will respond successfully to conventional orthodontic therapy and which patients may require combined orthodontic therapy and orthognathic surgery. In addition, since hyoid bone position is important to the maintenance of airway patency, hyoid position may be related to airway problems, such as Obstructive Sleep Apnea (OSA) and could be an early diagnostic feature of this syndrome. Future studies, possibly using software such as video fluoroscopy, depicting movement of the hyoid during swallowing may also lead to evaluation of swallowing as an etiological factor in malocclusion.

\section{Conclusions}

1) In hyperdivergent subjects, the posterior aspect of the hyoid is located 1.3 $\mathrm{mm}$ more inferior and $3.2 \mathrm{~mm}$ more posterior than in normodivergent subjects, while there is no difference in vertical position of the anterior surface of the hyoid.

2) The antegonial notch is $0.6 \mathrm{~mm}$ deeper in hyperdivergent patients than in normodivergent patients.

3) In males, the posterior aspect of the hyoid is positioned more inferiorly by $8.5 \mathrm{~mm}$, while the anterior surface is located $9.0 \mathrm{~mm}$ lower than in female subjects.

4) In males, the hyoid axis is more steeply inclined than in females by 4.4 degrees.

5) The antegonial notch is $0.6 \mathrm{~mm}$ deeper in males than in females.

6) There are statistical differences in hyoid bone position in hyperdivergent subjects compared to normodivergent subjects and in males compared to females. Therefore, the Null hypothesis is rejected.

\section{Conflicts of Interest}

The authors declare no conflicts of interest regarding the publication of this paper.

\section{References}

[1] Buschang, P.H., Sankey, W. and English, J.D. (2002) Early Treatment of Hyperdivergent Open-Bite Malocclusions. Seminars in Orthodontics, 8, 130-140. https://doi.org/10.1053/sodo.2002.125432

[2] Tumpkin, J. and Kudlick, E. (2014) The Hyoid Bone Position in Mouth Breathers: Cross-Sectional Study. Contemporary Clinical Dentistry, 5, 187-189.

[3] Buschang, P.H., Jacob, H. and Carrillo, R. (2013) The Morphological Characteristics, Growth, and Etiology of the Hyperdivergent Phenotype. Seminars in Orthodontics, 19, 212-226. https://doi.org/10.1053/j.sodo.2013.07.002

[4] Singer, C.P., Mamandras, A.H. and Hunter, W.S. (1987) The Depth of the Mandi- 
bular Antegonial Notch as an Indicator of Mandibular Growth Potential. American Journal of Orthodontics and Dentofacial Orthopedics, 91, 117-124. https://doi.org/10.1016/0889-5406(87)90468-9

[5] Bibby, R.E. and Preston, C.B. (1981) The Hyoid Triangle. American Journal of Orthodontics, 80, 92-97. https://doi.org/10.1016/0002-9416(81)90199-8

[6] Cangialosi, T. (2015) Chapter 19. Vertical Dimension and Anterior Open Bite. In: Mosby's Orthodontic Review, 2nd Edition, Elsevier, Amsterdam, 250-262.

[7] Converse, J.M., Coccaro, P.J., Becker, M. and Wood-Smith, D. (1973) On Hemifacial Microsomia. The First and Second Branchial Arch Syndrome. Plastic and Reconstructive Surgery, 5, 268-279. https://doi.org/10.1097/00006534-197303000-00005

[8] Isaacson, J.R., Isaacson, R.J., Speidel, T.M. and Worms, F.W. (1971) Extreme Variation in Vertical Facial Growth and Associated Variation in Skeletal and Dental Relations. The Angle Orthodontist, 41, 219-229.

[9] Grant, L.E. (1959) A Radiographic Study of Hyoid Bone Position in Angle's Class I, II, and III Malocclusions. Master's Thesis, University of Missouri, Kansas City.

[10] Urzal, V., Braga, A.C. and Ferreira, A.P. (2014) Hyoid Bone Position and Vertical Skeletal Pattern-Open Bite/Deep Bite. Oral Health and Dental Management, 13, 341-347.

[11] King, E.W. (1952) A Roentgenographic Study of Pharyngeal Growth. The Angle Orthodontist, 22, 23-37.

[12] Ingervall, B., Carlsson, G.E. and Helkimo, M. (1990) Change in Position of Hyoid Bone with Mandibular Positions. Acta Odontologica Scandinavica, 28, 337-361. https://doi.org/10.3109/00016357009032039

[13] Gustavsson, U., Hansson, G., Homqvist, A. and Lundberg, M. (1972) Hyoid Bone Position in Relation to Head Posture. Swedish Dental Journal, 65, 423-430.

[14] Adamidis, I.P. and Spyropoulos, M. (1992) Hyoid Bone Position and Orientation in Class I and Class III Malocclusions. American Journal of Orthodontics and Dentofacial Orthopedics, 101, 308-312. https://doi.org/10.1016/S0889-5406(05)80323-3

[15] Zheng, L., Jahn, J. and Vasavada, A.N. (2012) Sagittal Plane Kinematics of the Adult Hyoid Bone. Journal of Biomechanics, 45, 531-536. https://doi.org/10.1016/j.jbiomech.2011.11.040

[16] Bench, R.W. (1963) Growth of the Cervical Vertebrae as Related to Tongue, Face, and Denture Behavior. American Journal of Orthodontics, 49:183-214. https://doi.org/10.1016/0002-9416(63)90050-2

[17] Tsai, H.H., Ho, C.Y., Lee, P.L. and Tan, C.T. (2009) Sex differences in anthropometric and cephalometric characteristics in the severity of obstructive sleep apnea syndrome. American Journal of Orthodontics and Dentofacial Orthopedics, 135, 155-164. https://doi.org/10.1016/j.ajodo.2008.10.001

[18] Tallgren, A. and Solow, B. (1987) Hyoid Bone Position, Facial Morphology and Head Posture in Adults. European Journal of Orthodontics, 9, 1-8.

https://doi.org/10.1093/ejo/9.1.1

[19] Pae, E.K., Lowe, A.A., Sasaki, K., Price, C., Tsuchiya, M. and Fleetham, J.A. (1994) A Cephalometric and Electromyographic Study of Upper Airway Structures in the Upright and Supine Positions. American Journal of Orthodontics and Dentofacial Orthopedics, 106, 52-59. https://doi.org/10.1016/S0889-5406(94)70021-4

[20] Bibby, R.E. (1984) The Hyoid Bone Position in Mouth Breathers and TongueThrusters. American Journal of Orthodontics, 85, 431-433. 
https://doi.org/10.1016/0002-9416(84)90164-7

[21] Durzo, C.A. and Brodie, A.G. (1962) Growth Behaviour of the Hyoid Bone. The Angle Orthodontist, 32, 193-204.

[22] Athanasiou, A.E., Toutountzakis, N., Mavreas, D., Ritzau, M. and Wenzel, A. (1991) Alteration of Hyoid Bone Position and Pharyngeal Depth and Their Relationship after Surgical Correction of Mandibular Prognathism. American Journal of Orthodontics and Dentofacial Orthopedics, 100, 259-265.

https://doi.org/10.1016/0889-5406(91)70063-3

[23] Malkoc, S., Usumez, S., Nur, M. and Donaghy, C.E. (2005) Reproducibility of Airway Dimensions and Tongue and Hyoid Positions on Lateral Cephalograms. American Journal of Orthodontics and Dentofacial Orthopedics, 128, 513-516. https://doi.org/10.1016/j.ajodo.2005.05.001

[24] Subtelny, J.D. and Sakuda, M. (1964) Open-Bite: Diagnosis and Treatment. American Journal of Orthodontics and Dentofacial Orthopedics, 50, 337-358. https://doi.org/10.1016/0002-9416(64)90175-7

[25] Jena, A.K. and Duggal, R. (2011) Hyoid Bone Position in Subjects with Different Vertical Jaw Dysplasias. The Angle Orthodontist, 81, 81-85.

https://doi.org/10.2319/092208-491.1

[26] Haralabikis, N.B., Toutountzakis, N.M. and Yiagtzis, S.C. (1993) The Hyoid Bone Position in Adult Individuals with Openbite and Normal Occlusion. European Journal of Orthodontics, 15, 265-271. https://doi.org/10.1093/ejo/15.4.265

[27] Joseph, A.A., Elbaum, J., Cisneros, G.J. and Eisig, S.B. (1998) A Cephalometric Comparative Study of the Soft Tissue Airway Dimensions in Persons with Hyperdivergent and Normodivergent Facial Patterns. Journal of Oral and Maxillofacial Surgery, 56, 135-139. https://doi.org/10.1016/S0278-2391(98)90850-3

[28] Opdebeeck, H., Bell, W.H., Eisenfeld, J. and Mishelevich, D. (1978) Comparative Study between the SFS and LFS Rotation as a Possible Morphogenic Mechanism. American Journal of Orthodontics, 74, 509-521. https://doi.org/10.1016/0002-9416(78)90026-X

[29] Arslan, S.G., Dildes, N. and Kama, J.D. (2014) Cephalometric Investigation of First Cervical Vertebrae Morphology and Hyoid Position in Young Adults with Different Sagittal Skeletal Patterns. The Scientific World Journal, 2014, Article ID: 159784. https://doi.org/10.1155/2014/159784

[30] Cangialosi, T.J. (1984) Skeletal Morphologic Features of Anterior Open Bite. American Journal of Orthodontics, 85, 28-36. https://doi.org/10.1016/0002-9416(84)90120-9

[31] Andersen, W.S. (1963) The Relationship of the Tongue-Thrust Syndrome to Maturation and Other Factors. American Journal of Orthodontics and Dentofacial Orthopedics, 49, 264-275. https://doi.org/10.1016/0002-9416(63)90003-4

[32] Xiao, D., Gao, H. and Ren, Y. (2011) Craniofcial Morphological Characeristics of Chinese Adults with Normal Occlusion and Different Skeletal Divergence. European Journal of Orthodontics, 33, 198-204. https://doi.org/10.1093/ejo/cjq064

[33] Rana, T., Khanna, R., Tikku, T. and Schan, K. (2012) Relationship of Maxilla to Cranial Base in Different Facial Types-A Cephalometric Evaluation. Journal of Oral Biology and Craniofacial Research, 2, 30-35. https://doi.org/10.1016/S2212-4268(12)60008-6

[34] Nahoum, H.I. (1971) Vertical Proportions and the Palatal Plane in Anterior Open Bite. American Journal of Orthodontics, 59, 273-282.

https://doi.org/10.1016/0002-9416(71)90100-X 
[35] Nahoum, H.I. (1975) Anterior Open-Bite: A Cephalometric Analysis and Suggested Treatment Procedures. American Journal of Orthodontics, 67, 513-521. https://doi.org/10.1016/0002-9416(75)90297-3

[36] Lambrechts, A.H., Harris, A.M., Rossouw, P.E. and Stander, I. (1996) Dimensional Differences in the Craniofacial Morphologies of Groups with Deep and Shallow Mandibular Antegonial Notching. The Angle Orthodontist, 66, 265-272.

[37] Davidovitch, M., Eleftheriadi, I., Kostaki, A. and Shpack, N. (2016) The Use of Bjork's Indications of Growth for Evaluation of Extremes in Skeletal Morphology. European Journal of Orthodontics, 8, 555-562. https://doi.org/10.1093/ejo/cjv084

[38] Mangla, R., Singh, N., Dua, V., Padmanabhan, P. and Khanna, M. (2011) Evaluation of Mandibular Morphology in Different Facial Types. Contemporary Clinical Dentistry, 2, 200-206. https://doi.org/10.4103/0976-237X.86458

[39] Tomer, G. and Kishnani, R. (2011) Correlation of Antegonial Notch Depth with Craniofacial Morphology-A Cephalometric and Electromyographic Study. Journal of Pierre Fauchard Academy (India Section), 25, 163-171.

https://doi.org/10.1016/S0970-2199(11)53006-1 